Brit. Heart f., 1969, 31, 629.

\title{
Value of Vectorcardiogram in Diagnosis of Posterior and Inferior Myocardial Infarctions
}

\author{
P. TOUTOUZAS $\star$, P. HUBNER, G. SAINANI, AND J. SHILLINGFORD
}

From the M.R.C. Cardiovascular Research Unit, Royal Postgraduate Medical School, London W.12

The scalar and vectorcardiographic criteria for the diagnosis of myocardial infarction are well established (Goldberger, 1953; Massie and Walsh, 1960; Tandowsky, 1968). In many cases of myocardial infarction, the clinical history, diagnostic transient rise of serum enzymes, and a clear-cut pattern of infarction on the electrocardiogram make the diagnosis of infarction unequivocal. With this type of patient the vectorcardiogram, though of interest, will not be of further diagnostic value, as the diagnosis is not in doubt. There are cases, however, especially after posterior or inferior infarction, where the electrocardiographic changes of infarction may be only minimal or equivocal. The purpose of this paper is to assess the value of the vectorcardiogram in offering support to the electrocardiogram in the diagnosis of infarction at these sites.

\section{SUBJECTS AND METHODS}

Studies were made on 100 patients with the clinical diagnosis of myocardial infarction, with transient diagnostic rise of serum lactate dehydrogenase, and with electrocardiographic changes of posterior or inferior infarction. Changes of coexistent anterior infarction did not exclude patients with inferior infarction from the series. The interval between the acute episode and time of study ranged between 2 weeks and 3 years.

An electrocardiogram and a vectorcardiogram, on the same day, were made for each patient. The electrocardiogram was a standard 12-lead form, with the addition of V4R and V7 chest leads. The vectorcardiogram, using the Frank electrode system (Frank, 1956), was obtained for frontal, horizontal, and left sagittal planes on a Cambridge Multichannel Recorder. $\dagger$ Recordings were made at standardizations of 1 millivolt $=1,2,4$,

Received February 19, 1969.

* In receipt of a grant from the Wellcome Trust.

t Cambridge Instrument Company, London. and $6 \mathrm{~cm}$. $0.005 \mathrm{sec}$. was the time interval between the markers whose shape showed the direction of the loop.

The electrocardiograms were divided into those showing classical or clear-cut criteria and those showing only equivocal changes for posterior, and/or inferior myocardial infarction.

Classical electrocardiographic criteria of infarction used were those of Goldberger (1953), Massie and Walsh (1960), and World Health Organization (1959).

(1) Posterior infarction: (a) $R / S$ ratio in V4R and V1 greater than 1 or (b) RSR pattern in V4R and V1 of less than 0.1 sec. duration.

(2) Inferior infarction: $Q$ wave of $0.03 \mathrm{sec}$. or more duration, with a depth 25 per cent or more of a succeeding $\mathbf{R}$ wave.

Equivocal electrocardiographic changes of infarction consisted of the following.

(1) Posterior infarction: (a) Tall "tented" $\mathrm{T}$ waves $V 1, V 2$, and V3, or (b) $R$ waves present in V4R, V1, and $V 2$, but $R / S$ ratio less than 1 in $V 4 R$ and $V 1$, or greater than 1 in $\mathrm{V} 2$.

(2) Inferior infarction: (a) $\mathrm{T}$ inversion in II, III, and aVF without a $Q$ wave and/or (b) $r S$ pattern in II, III, and aVF.

The vectorcardiographic QRS loop criteria of infarction were those of Hugenholtz, Forkner, and Levine (1961) and Tandowsky (1968):

(1) Posterior infarction: increased magnitude and duration of the anterior forces.

(2) Inferior infarction: (a) Superior and leftward displacement of $0.025 \mathrm{sec}$. vector or (b) superior displacement of maximal $Q R S$ vector above $+20^{\circ}$ in the frontal plane, with initial clockwise rotation on this plane.

Finally, the vectorcardiograms of 50 normal people were studied to establish the normal QRS loop patterns obtained with the Cambridge Multichannel Recorder. There were 37 men and 13 women, whose ages ranged from 22 to 67 . Each person had a negative history for heart disease, had normal blood pressure, and had a normal cardiovascular system examination, chest $x$-ray, 629 


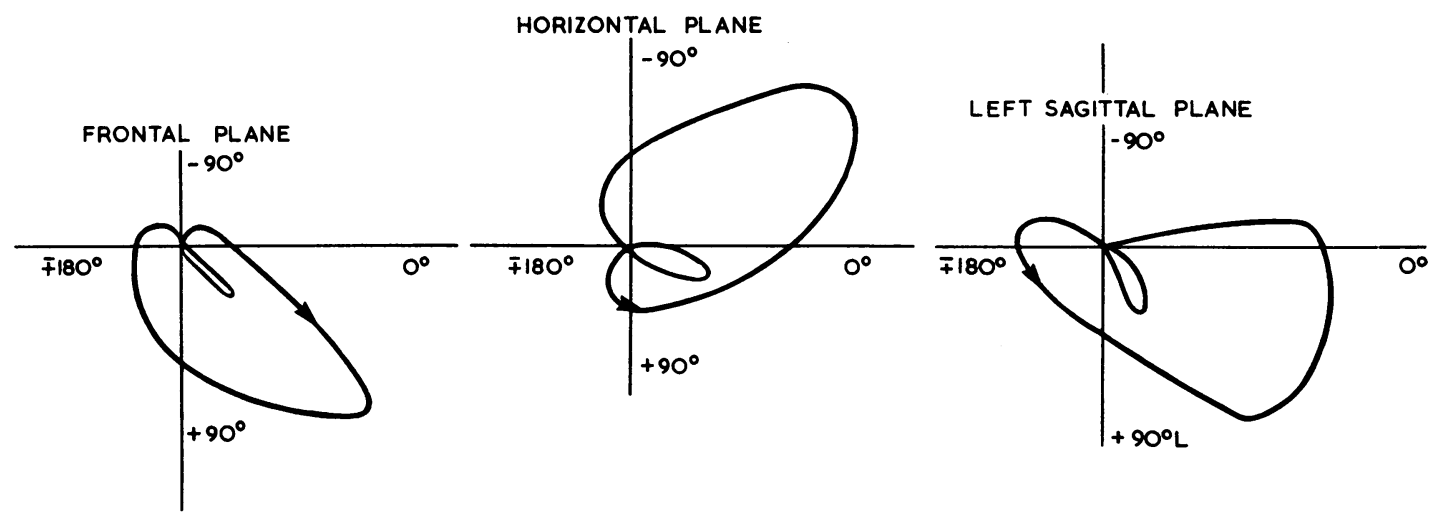

FIG. 1.-Normal QRS and T loops on horizontal, frontal, and left sagittal planes, derived from the average of 50 normal subjects.

\section{Results}

I: Normal Subjects. The average QRS loop in the 3 planes obtained from the 50 normal subjects is shown in Fig. 1. The loops correspond well with those of other reported normal series using different equipment (Draper et al., 1964; Lyon and Belletti, 1968). On this basis, the criteria established for infarction on other equipment are considered to be valid for vectorcardiograms from the Cambridge Multichannel Recorder.

II: Patients. Of the 100 patients, 86 showed a classical electrocardiographic pattern of posterior, and/or inferior infarction, and this finding was confirmed in each case on the QRS loop pattern of the vectorcardiogram. Fourteen patients, though showing equivocal changes of infarction on the electrocardiogram, had definite diagnostic features of infarction on the vectorcardiogram. The vectorcardiogram showed 9 posterior and 5 inferior infarctions in these patients. Fig. 2-5 are electrocardiograms and vectorcardiograms of representative examples of these patients.

The following case reports are of 2 such patients whose electrocardiograms showed only equivocal changes, but whose vectorcardiograms were diagnostic of infarction.

\section{CASE REPORTS}

Case 1. This patient was admitted with a classical history of myocardial infarction. Apart from mild pulmonary oedema on the second day, which cleared after diuretic therapy, recovery was uncomplicated. The serum lactic dehydrogenase rose to a maximum of 1400 I.U. ( $65 \%$ heat stable: normal $=70-150$ I.U., $30-$ $65 \%$ heat stable) on the third day, suggestive of a large infarction. However, serial electrocardiograms showed only minimal or equivocal changes. The electrocardiogram and vectorcardiogram on the 15 th day after infarc- tion are shown in Fig. 2. The electrocardiogram shows a prominent $R$ wave in $V 2$, with ST segment depression in leads I, II, V2 to V5, and T wave inversion or flattening in V6, V7, I, and aVL. The vectorcardiogram shows the distinctive pattern for posterior infarction, with displacement of the QRS loop anteriorly in the horizontal and sagittal planes; the anterior forces are greater than the posterior ones. The vectorcardiogram clearly showed the presence of posterior infarction despite only equivocal changes on the electrocardiogram.

Case 2. This patient also sustained a myocardial infarction, with a classical history, and a maximum rise of serum lactic dehydrogenase to 310 I.U. (70\% heat stable) on the 3rd day. Recovery was uneventful. The electrocardiogram and vectorcardiogram 24 days after the onset of infarction are shown in Fig. 5. The electrocardiogram was only equivocal for infarction, showing rS complex in aVF and rSr complex in III without $T$ wave inversion. The vectorcardiogram showed the diagnostic pattern for inferior infarction, with displacement of most of the QRS loop superiorly, to give maximal QRS loop vector of $-10^{\circ}$ in the frontal plane. The initial $r$ wave of the inferior lead of the electrocardiogram is explained by the QRS loop; the forces are initially inferiorly directed, but subsequently they turned upwards, so that the vectorcardiographic criteria for inferior infarction are satisfied.

\section{Discussion}

This study has shown that in a small percentage $(14 \%)$ of patients with a positive history and diagnostic transient serum enzyme rise of myocardial infarction, the vectorcardiogram was of practical supportive value to an electrocardiogram showing only minor or equivocal changes of inferior or posterior infarction. With the vectorcardiogram the diagnosis of infarction could be made with greater certainty.

It is well established that the classical pattern of infarction may be absent after myocardial infarction; 

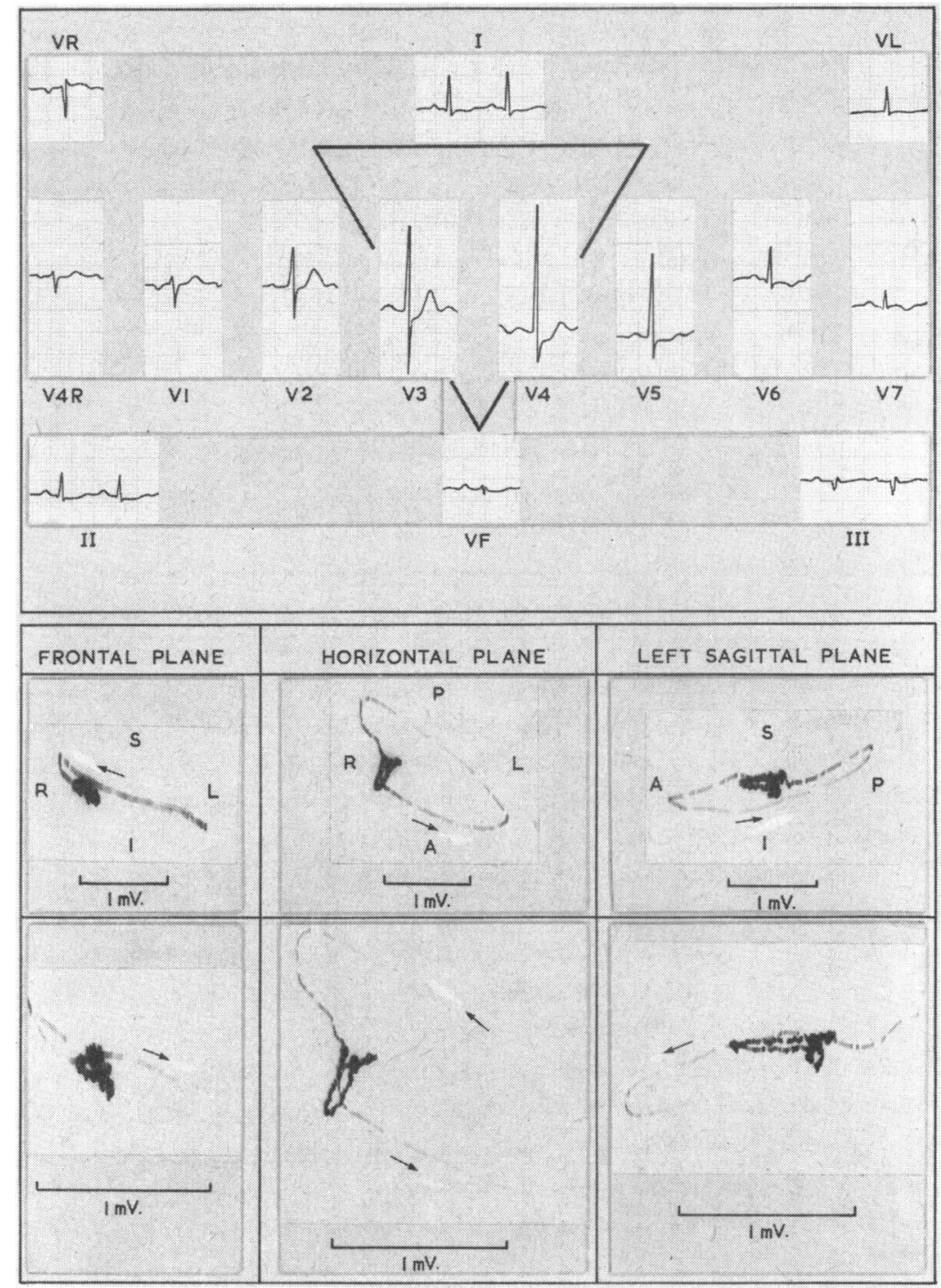

FIG. 2.-Vectorcardiogram and electrocardiogram of a patient with posterior infarction (Case 1, see text). Electrocardiographic changes of infarction are minimal. The vectorcardiographic changes of anterior displacement of the QRS loop are diagnostic of posterior infarction. The diagram illustrates the changes of the QRS loop (continuous line) from the normal loop (interrupted line) on the frontal plane. Arrows indicate the direction of the loops.

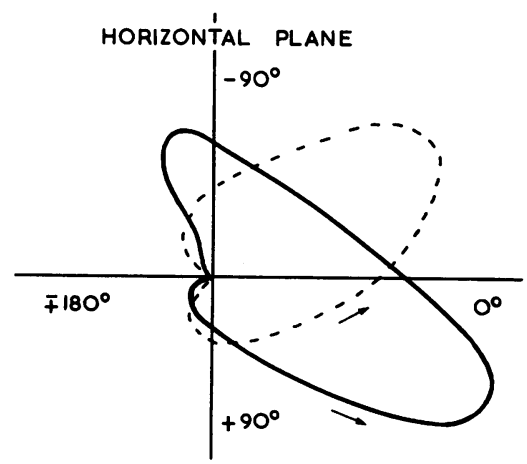



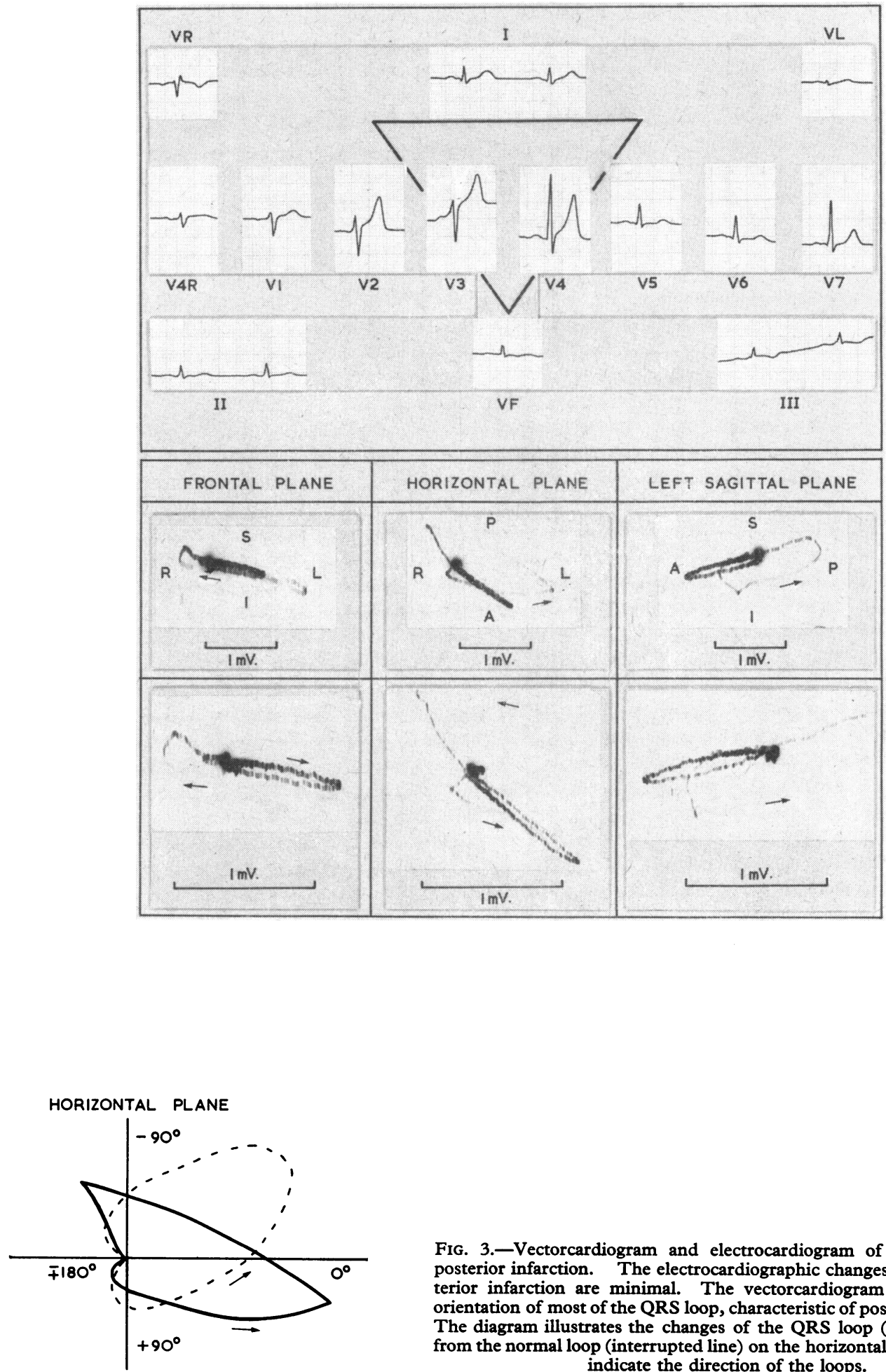

Fig. 3.-Vectorcardiogram and electrocardiogram of a patient with posterior infarction. The electrocardiographic changes suggesting posterior infarction are minimal. The vectorcardiogram shows anterior orientation of most of the QRS loop, characteristic of posterior infarction. The diagram illustrates the changes of the QRS loop (continuous line) from the normal loop (interrupted line) on the horizontal plane. Arrows indicate the direction of the loops. 


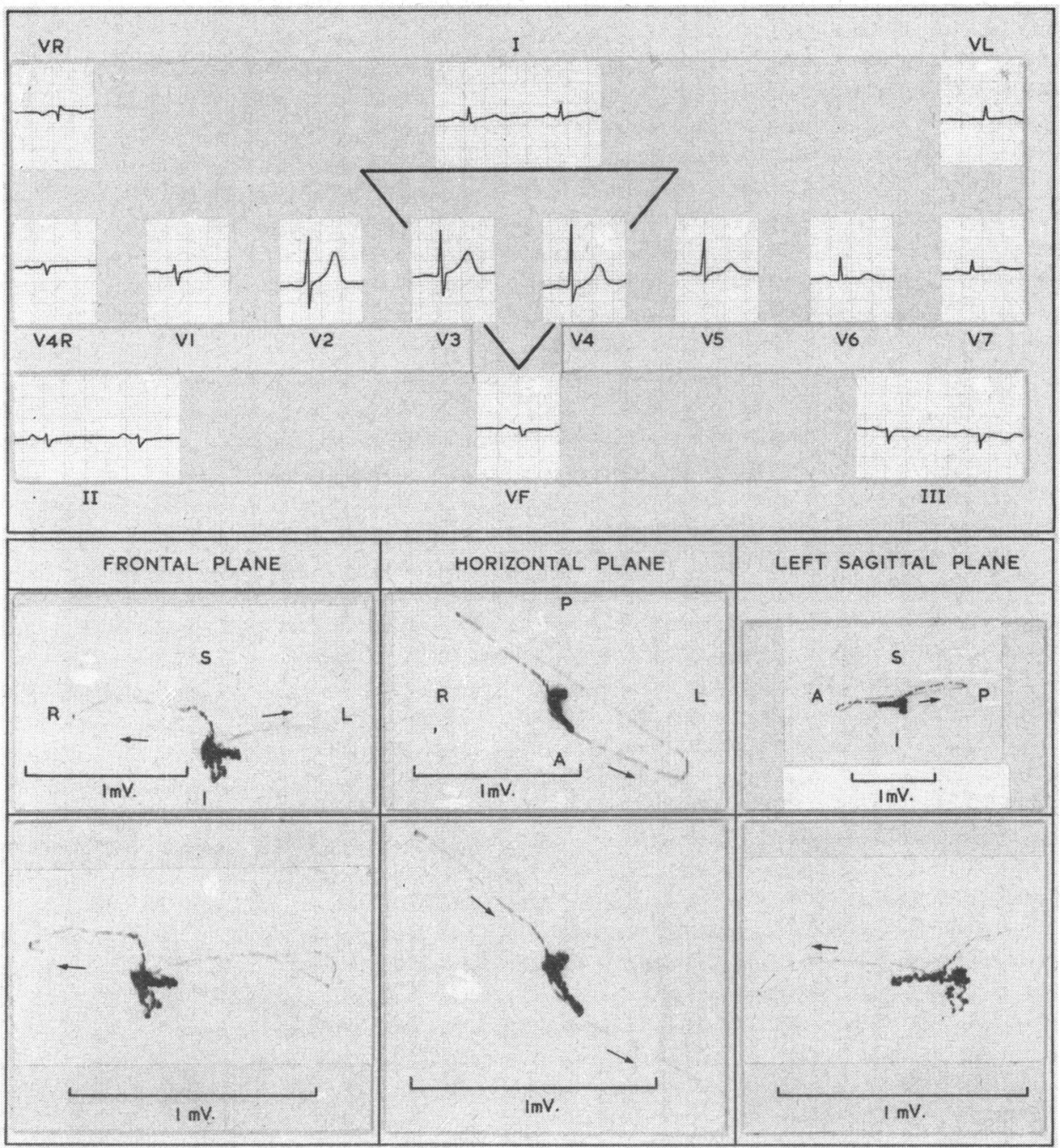

Fig. 4.-Vectorcardiogram and electrocardiogram of a patient with inferior infarction. The electrocardiogram shows $\mathrm{rS}$ complexes with $\mathrm{T}$ inversion in III and aVF. There is no $Q$ wave of infarction. The vectorcardiogram shows superior displacement of the QRS loop, diagnostic of inferior infarction. The diagram illustrates the changes of the QRS loop (continuous line) from the normal loop (interrupted line) on the frontal plane. Arrows alongside loops indicate their directions: arrow on loop indicates the superiorly directed 0.025 vector. Letter A points to the inferiorly orientated early QRS forces $(0.01$ vector), recorded as an initial $r$ wave on leads II, III, and aVF of the

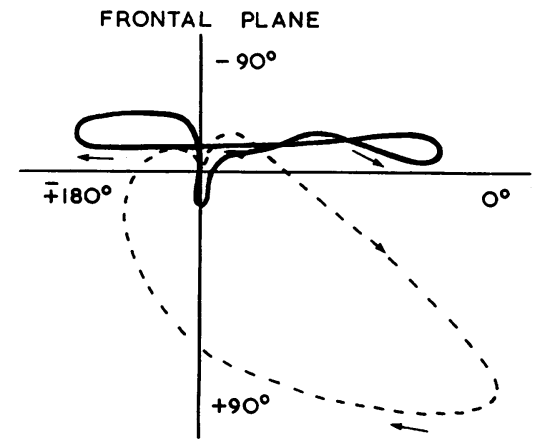



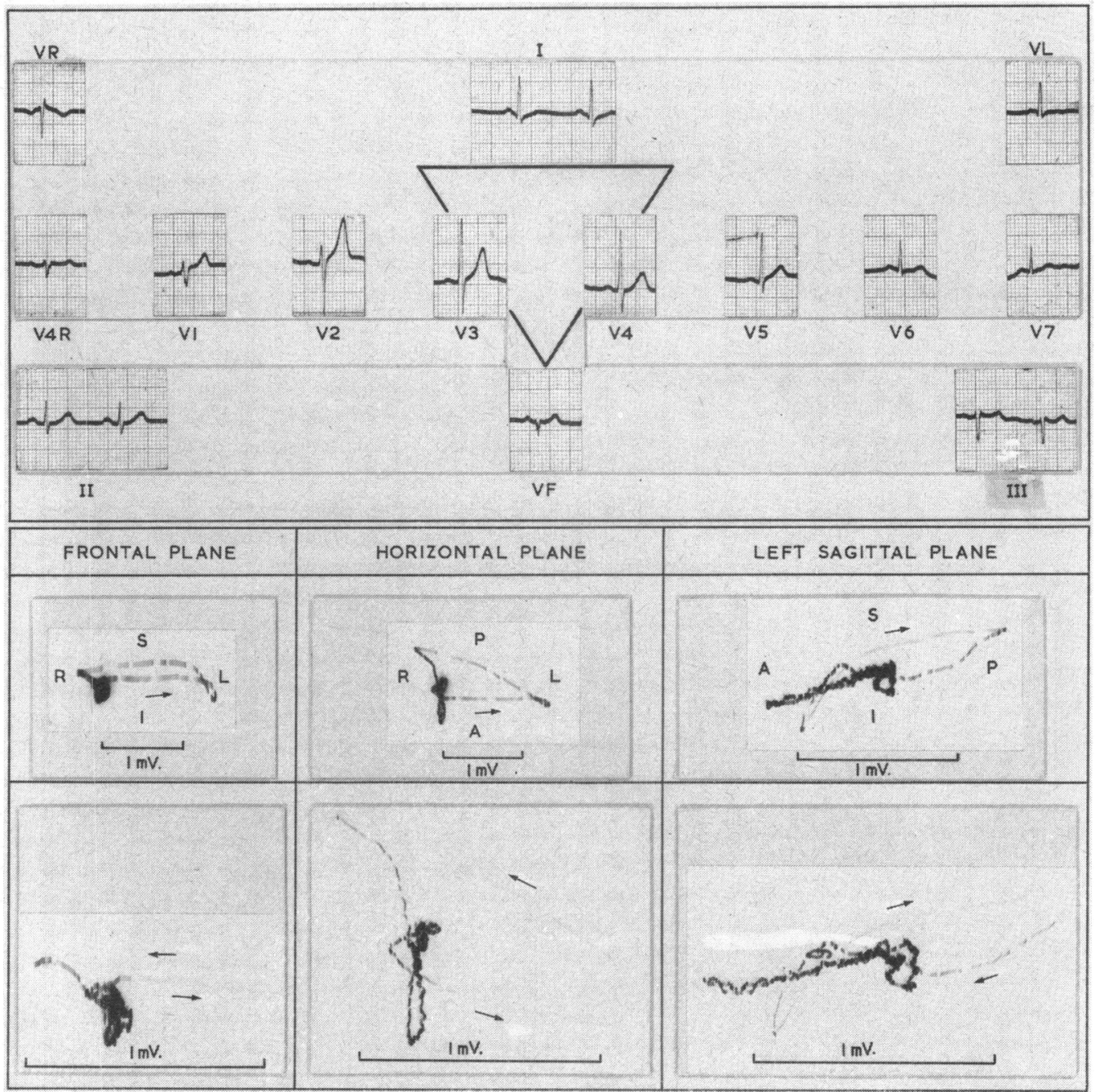

FRONTAL PLANE

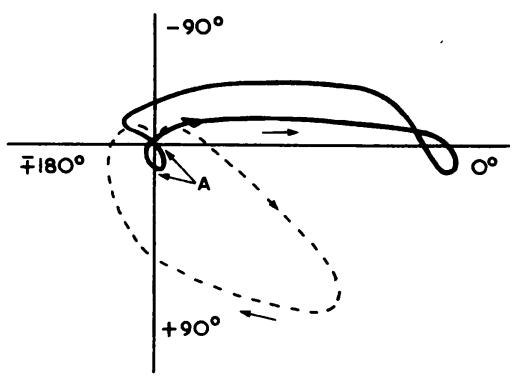

Fig. 5.-Vectorcardiogram and electrocardiogram of a patient with inferior infarction (Case 2, see text). The electrocardiogram shows a slurred $\mathrm{rS}$ wave in $\mathrm{aVF}$ and an $\mathrm{rSr}$ complex in III, without $\mathrm{T}$ wave inversion. These changes are only equivocal for inferior infarction. The vectorcardiogram shows superior displacement of the QRS loop, characteristic of inferior infarction. The diagram illustrates the changes of the QRS loop (continuous line) from the normal loop (interrupted line) on the frontal plane. Arrows alongside loops indicate their direction; arrow on loop indicates the superiorly directed 0.025 vector. Letter A points to the inferiorly orientated early QRS forces $(0.01$ vector), recorded as an initial $r$ wave on the inferior leads of the electrocardiogram. 
this is particularly true for the absence of a pathological $Q$ wave (in the inferior leads) after inferior infarction (Burch et al., 1958; Johnson et al., 1959). The electrocardiographic changes may not yet have developed in the early stages after infarction (Short, 1968); subsequently they may diminish so that eventually the electrocardiogram may return completely to normal (Cox, 1967). At these two extremes the vectorcardiogram is likely to be normal too. However, when after inferior infarction there are equivocal or minor changes on the electrocardiogram such as $T$ wave changes or an rS complex instead of a $Q$ wave, the vectorcardiogram may show a diagnostic pattern of infarction.

Posterior infarction was the site where a vectorcardiogram most frequently demonstrated an infarction from an electrocardiogram which was only equivocal. The widely used electrocardiographic criterion for the diagnosis of posterior infarction, of an $R / S$ ratio in V4R and V1 greater than 1, is not infrequently absent. When this criterion is satisfied, the site of infarction as shown by the vectorcardiogram is more usually postero-infero-lateral (Toutouzas and Hubner, in preparation).

The reason why the vectorcardiogram may show infarction while the electrocardiogram shows only minor changes, lies in the relative ability to study ventricular depolarization by the two methods. The vectorcardiogram allows a much better study and analysis of the size, duration, and rate of inscription of the QRS forces than the standard electrocardiogram. The vectorcardiogram can be magnified so that 1 millivolt is represented by up to $6 \mathrm{~cm}$. on the record. Some of these disadvantages of the standard electrocardiogram may be overcome by high speed recordings.

It is suggested from this study that for the majority of patients the electrocardiogram, particularly with serial records, will show an unequivocal pattern of infarction and be adequate to establish the diagnosis of posterior or inferior infarction. The vectorcardiogram will be of interest but will not add anything further to the diagnosis. In a small proportion of patients, perhaps 10-15 per cent, where electrographic changes are minimal or equivocal, the vectorcardiogram may show clearly a myocardial infarction. The vectorcardiogram will supply supportive evidence to the electrocardiogram and give strength to the diagnosis of myocardial infarction. This role will perhaps be greater in the Outpatient Department for patients presenting without a clear history of ischaemic heart disease and where the electrocardiogram shows equivocal changes of infarction.

\section{SUMMARY}

A study was made of 100 patients with posterior or inferior infarction to assess the ability of the vectorcardiogram in supporting the electrocardiographic diagnosis of infarction. Of these patients, 86 were found to have clear-cut evidence of infarction on the electrocardiogram; the vectorcardiogram, though of interest, did not add anything further to the diagnosis. Fourteen patients had equivocal changes of infarction on the electrocardiogram but a definite pattern of infarction on the vectorcardiogram. The vectorcardiogram is of diagnostic value and able to supplement the electrocardiogram in a small proportion of patients after myocardial infarction, whose changes on the electrocardiogram are minimal or equivocal.

We acknowledge with pleasure the technical assistance given by Miss Jean Powell and Mr. Peter Burgess.

\section{REFERENCES}

Burch, G. E., Horan, L. G., Ziskind, J., and Cronvich, J. A. (1958). A correlative study of postmortem, electrocardiographic, and spatial vectorcardiographic data in myocardial infarction. Circulation, 18, 325.

Cox, C. J. Burns (1967). Return to normal of the electrocardiogram after myocardial infarction. Lancet, 1, 1194.

Draper, H. W., Peffer, C. J., Stallmann, F. W., Littmann, D., and Pipberger, H. V. (1964). The corrected orthogonal electrocardiogram and vectorcardiogram in 510 normal men (Frank lead system). Circulation, 30, 853.

Frank, E. (1956). An accurate, clinically practical system for spatial vectorcardiography. Circulation, 13, 737.

Goldberger, E. (1953). Unipolar Lead Electrocardiography and Vectorcardiography. Lea and Febiger, Philadelphia.

Hugenholtz, P. G., Forkner, C. E., and Levine, H. D. (1961). A clinical appraisal of the vectorcardiogram in myocardial infarction. II. The Frank system. Circulation, 24, 825.

Johnson, W. J., Achor, R. W. P., Burchell, H. B., and Edwards, J. E. (1959). Unrecognized myocardial infarction: a clinicopathologic study. Arch. intern. Med., $103,253$.

Lyon, A. F., and Belletti, D. A. (1968). The Frank vectorcardiogram in normal men. Norms derived from visual and manual measurement of 300 records. Brit. Heart f., 30, 172.

Massie, E., and Walsh, T. J. (1960). Clinical Vectorcardiography and Electrocardiography. Year Book Publishers, Chicago.

Short, D. (1968). Value and limitations of electrocardiogram in diagnosis of slight and subacute coronary attacks. Brit. med. F., 4, 673.

Tandowsky, R. M. (1968). The Oscillometric Vectorcardiogram. Charles C. Thomas, Springfield, Illinois.

World Health Organization (1959). Hypertension and coronary heart disease: Classification and criteria for epidemiological studies. Wld Hlth Org. tech. Rep. Ser., No. 168. 